\title{
TRANSRECTAL ULTRASOUND PROSTATE BIOPSY TRACKING WITH EFFICIENT AND ACCURATE DEFORMATION ESTIMATION
}

\author{
Michael Baumann ${ }^{1,2}$, Pierre Mozer ${ }^{3}$, Vincent Daanen ${ }^{2}$ and Jocelyne Troccaz ${ }^{1 *}$ \\ ${ }^{1}$ Université J.Fourier, TIMC laboratory, Grenoble, France; CNRS, UMR 5525. \\ ${ }^{2}$ Koelis SAS, 5. av. du Grand Sablon, 38700 La Tronche, France. \\ ${ }^{3}$ La Pitié-Salpêtrière hospital, urology dpt, 75651 Paris Cedex 13, France.
}

\begin{abstract}
Prostate cancer is the most frequent cancer for men and the second cause of cancer death. Confirmation of cancer requires transrectal prostate biopsies. Tracking systems for transrectal prostate biopsies make it possible to generate biopsy distribution maps for intra- and post-interventional quality control, 3D visualisation of histological results for treatment planning, and to guide clinicians toward nonultrasound (US) targets. In this paper, a 3D US based tracking system for fast and accurate estimation of nonlinear prostate tissue deformation is proposed. Linear elasticity and inverse consistency a priori models are used to improve performance. A variant of the SSD image distance measure is proposed for filtering of local intensity shifts, which are frequent in US images. System accuracy was evaluated to $0.83 \pm 0.54 \mathrm{~mm}$ using point fiducial on 295 US volumes from 18 patients.
\end{abstract}

Index Terms - 3D US-based organ tracking, prostate biopsies, prostate tracking, deformation tracking

\section{INTRODUCTION}

The only definitive way to confirm a prostate cancer suspicion is the histological examination of tissue samples. Conventional prostate biopsies are performed under 2D US control using an 18 gauge puncture needle. The gland can be accessed either by the transperineal path, or the less invasive transrectal path, which is the current clinical standard. For transrectal access, the needle is mechanically aligned with the US image plane via a rigidly attached tubular needle guide, which makes it possible to visualize the needle trajectory in the US images.

Most tumors are of isoechogenic nature, i.e. they are not visually detectable in the US image. The biopsy sites are therefore chosen using a systematic protocol which typically requires the sampling of 10 to 12 biopsies. The fact that the biopsy targets cannot be identified on the control US images represents a severe weakness of prostate biopsies since there

\footnotetext{
*Thanks to the Agence Nationale de la Recherche (TecSan project, France), the French Ministry of Health (PHRC program, France) and to Koelis S.A.S. (France) for funding.
}

is no guarantee that an existing tumor will be sampled. This leaves the clinician in a dilemma when the cancer hypothesis cannot be discarded: his only option is to repeat the biopsies. Furthermore, it is challenging for the clinician to follow the systematic protocol accurately, due to the fact that the probe is continually moved for needle placement; a constant visual reference is lacking. This may lead to undersampling of parts of the gland. Moreover, the exact sampling location is unknown after acquisition, which makes it difficult to reuse histological results for locally adapted treatments. MR and spectro-MR imaging is more sensitive to prostate carcinoma, but biopsy acquisition under MR control requires special instruments. Also, it is difficult to imagine the generalization of MR biopsies because of the high cost and lack of availability of MR systems - note that more than two million prostate biopsies are performed every year in the USA and Europe.

Baumann et al. [1] and Xu et al. [2] simultaneously proposed computer assisted biopsies to address these problems. The idea is to acquire a reference US volume of the prostate prior to the intervention. During the intervention, the stream of US control images is registered with this reference volume, thus allowing for projection of planning data into the control images and, conversely, the projection of the biopsy trajectories into the reference volume. Predefined targets can consist of suspicious lesions detected in an MR image after registration with the reference volume, they can stem from statistical cancer distributions or they can be zones unsampled during a previous biopsy session.

Organ tracking via image registration is, however, not trivial. The patient is not under total anesthesia and experiences a certain discomfort and sometimes pain. It is frequent that he moves his pelvis and, hence, the organ relative to the operating room. In addition, the probe and thus the US beam moves with respect to the gland during needle positioning, and the organ will often be only partially viewed. Finally, the gland itself moves and gets deformed due to probe pressure, but also due to the activity of neighboring organs.

$\mathrm{Xu}$ et al. proposed magnetic probe tracking to follow 2D US beam movements with respect to the operating room, and to compensate small patient movements with image registra- 
tion [2]. Bax et al. replace the magnetic tracker by an encoded articulated arm that holds the probe to track the US beam, but they do not correct patient movements [3]. 2D US beam tracking based approaches have two severe draw-backs: first, significant pelvis movements lead to registration failure, even when combined with image registration. The reason is that 3D-2D image registration requires a fairly good initial position estimate for not to get stuck in a local minimum during image similarity optimization. If the system loses track, a freehand US volume has to be acquired again for the novel organ position, which is very time-consuming and seems impractical. Second, it is difficult to estimate organ deformations reliably, since the 2D images do not provide sufficient information. However, most prostate tumors grow in the peripheral zone of the gland, which is particularly exposed to deformations caused by probe head pressure. Deformations of $5 \mathrm{~mm}$ and more are frequent near the probe head. Baumann et al. address these draw-backs by using 3D US to obtain richer control images during the intervention [1]. Instead of using a US beam tracking device to initialize local image-based registration, they propose a cinematic model of endorectal probe movements to estimate anatomically plausible positions of the view-cone with respect to the gland. A cohort study on 47 patients showed that this method is not impaired by patient movements [4].

In this paper an extension of the 3D US tracking method is presented that estimates gland deformations. The challenge is to achieve a highly efficient deformation estimation algorithm that is sufficiently robust to be used in a clinical context. A registration framework is proposed that achieves both objectives using multi-resolution techniques, inverse consistency constraints and a specialized local image distance measure capable of handling low-frequency intensity changes often present in US images. The accuracy of the presented method is evaluated on clinical patient data.

\section{METHODS}

Image-based organ tracking requires robust and efficient transformation estimation techniques. A technique to achieve this goal is to use a highly pipelined registration system, where the degrees of freedom of the estimated transformation are successively increased. For the rigid 3D US based tracking of the prostate, a 3-step pipeline is used to determine the rigid transformation [1]. In this paper, we will add a fourth step for deformation estimation to the pipeline.

Image-based deformation estimation can be formulated as an optimization process of a local distance measure. Let $I_{1}, I_{2}: \mathbb{R}^{3} \rightarrow \mathbb{R}$ be images, $\varphi: \mathbb{R}^{3} \rightarrow \mathbb{R}^{3}$ the deformation function and the functional $\mathcal{D}\left[I_{1}, I_{2} ; \varphi\right]$ a measure of the distance between $I_{1}$ and $I_{2} \circ \varphi$. In contrast to parametric approaches that use basis functions to build the deformation function, we will follow a variational approach and define $\varphi(x)=x+u(x)$, where $u: \mathbb{R}^{3} \rightarrow \mathbb{R}^{3}$ is an arbitrary function.
A simple energy function to be minimized could be defined as

$$
\mathcal{E}\left[I_{1}, I_{2} ; \varphi\right]=\mathcal{D}\left[I_{1}, I_{2} ; \varphi\right]
$$

and the optimization process as

$$
\varphi^{*}=\underset{\varphi}{\arg \min }\left(\mathcal{E}\left[I_{1}, I_{2} ; \varphi\right]\right) .
$$

Straight-forward minimization of a distance measure yields in general poor results due to countless local minima, in particular in presence of noise, partial object occlusion and other imperfections in the image data. Unfortunately, US is a particularly noisy modality, which makes 3D US based deformation estimation vulnerable to local misregistrations. This problem can be addressed by integration of a priori models of the expected deformation. This can be done implicitly by adding further energy terms to the objective function. In this work, inverse consistency and elastic regularization energies are added.

\subsection{Inverse Consistency Constraints}

In non-linear image registration, the forward estimation that minimizes $\mathcal{E}\left[I_{1}, I_{2} ; \varphi\right]$ does in general not yield the inverse of the backward estimation that minimizes $\mathcal{E}\left[I_{2}, I_{1} ; \psi\right]$, i.e. $\varphi \circ \psi \neq I d$ with $I d: \mathbb{R}^{3} \rightarrow \mathbb{R}^{3}, x \mapsto x$. Introduction of Zhang's inverse consistency constraint [5]

$$
\mathcal{I}[\psi ; \varphi]=\int_{\Omega}\|\psi \circ \varphi-I d\|_{\mathbb{R}^{3}}^{2} d x
$$

as additional energy penalizes solutions that lead to inconsistent inverse transformations, where $\Omega \subset \mathbb{R}^{3}$ is the registration domain in image space. Estimation of the forward and the backward deformations is coupled by an alternating iterative optimization

$$
\begin{aligned}
\varphi^{k+1} & =\underset{\varphi}{\arg \min }\left(\mathcal{E}\left[I_{1}, I_{2} ; \varphi\right]+\mathcal{I}\left[\psi^{k} ; \varphi\right]\right), \\
\psi^{k+1} & =\underset{\psi}{\arg \min }\left(\mathcal{E}\left[I_{2}, I_{1} ; \psi\right]+\mathcal{I}\left[\varphi^{k} ; \psi\right]\right) .
\end{aligned}
$$

Concurrent estimation with mutual correction reduces the risk of local misregistrations.

\subsection{Elastic Regularization}

The deformation of the prostate caused by probe pressure is fully elastic, which justifies the introduction of the linearized elastic potential [6]

$$
\begin{aligned}
\mathcal{E}[\varphi] & =\mathcal{E}[u+I d] \\
& =\int_{\Omega} \frac{\mu}{4} \sum_{j, k=1}^{3}\left(\partial_{x_{j}} u_{k}+\partial_{x_{k}} u_{j}\right)^{2}+\frac{\lambda}{2}(\operatorname{div} u)^{2} d x
\end{aligned}
$$

as additional energy, where $\lambda$ and $\mu$ are the Lamé coefficients. 


\subsection{Image Distance Measure}

The image distance measure is the driving energy of the optimization process. Experiments on patient data have shown that the sum of squared distances (SSD) is a poor distance measure for noisy US images. Local intensity changes are frequent due to changing US beam angles with respect to the tissues and probe pressure variations. The more robust Pearson correlation coefficient (CC) requires the evaluation of a large neighborhood of every voxel pair to yield statistically significant results, which is incompatible with deep multi-resolution approaches that operate on very coarse levels.

We hence prefer an intermediate correlation model that filters low-frequency intensity shifts, i.e. we assume that $I_{1} \equiv$ $I_{2} \circ \hat{\varphi}+b$, where $\hat{\varphi}$ is the physical solution of the registration problem, and where $b: \mathbb{R}^{3} \rightarrow \mathbb{R}^{3}$ models a local intensity shift. The shift is estimated by

$$
b^{\sigma}[\varphi](x)=\left(I_{1}-I_{2} \circ \varphi\right) * \mathcal{G}_{\sigma}(x)
$$

where $\mathcal{G}: \mathbb{R}^{3} \rightarrow \mathbb{R}$ is a Gaussian with standard deviation $\sigma$. The image distance energy is then

$$
\mathcal{D}\left[I_{1}, I_{2} ; \varphi\right]=\int_{\Omega}\left(I_{1}(x)-I_{2}(\varphi(x))-b^{\sigma}[\varphi](x)\right)^{2} d x .
$$

The standard deviation $\sigma$ controls the frequency range of the high-pass filter. If $\sigma$ gets smaller, the cropped frequency range gets larger, and registration convergence rate decreases and may even stall if only high frequency noise like speckle is left. When used with a multi-resolution solver on a Gaussian pyramid (cf. next section), which implicitly performs a low-pass filtering of the intensity variations on coarse resolutions, this approach transforms to a band-pass filtering on varying frequency bands. In this configuration it is sufficient to chose relatively small standard deviations without risking registration inefficiencies.

\subsection{Solver}

Combination of the energy terms yields the alternating system

$$
\begin{aligned}
\varphi^{*} & =\underset{\varphi}{\arg \min }\left(\mathcal{D}\left[I_{1}, I_{2}, \varphi\right]+\mathcal{E}[\varphi]+\mathcal{I}[\psi ; \varphi]\right), \\
\psi^{*} & =\underset{\psi}{\arg \min }\left(\mathcal{D}\left[I_{2}, I_{1}, \psi\right]+\mathcal{E}[\psi]+\mathcal{I}[\varphi ; \psi]\right) .
\end{aligned}
$$

An iterative two-step minimization scheme is used to solve both objective functions. The Euler-Lagrange equations of Eqn. 9 and 10 are rewritten as a fixed point iteration

$$
\begin{aligned}
& \frac{\varphi^{k+1}-\varphi^{k}}{\Delta t}=\mathcal{L}\left[\varphi^{k}\right]+f_{\mathcal{D}}\left[I_{1}, I_{2} ; \varphi^{k}\right]+f_{\mathcal{I}}\left[\psi^{k} ; \varphi^{k}\right], \\
& \frac{\psi^{k+1}-\psi^{k}}{\Delta t}=\mathcal{L}\left[\psi^{k}\right]+f_{\mathcal{D}}\left[I_{2}, I_{1} ; \varphi^{k}\right]+f_{\mathcal{I}}\left[\varphi^{k} ; \psi^{k}\right],
\end{aligned}
$$

where $t \in \mathbb{R}$ controls the discretization granularity, and with the elliptic partial differential operator

$$
\mathcal{L}[\varphi]=\mathcal{L}[u+I d]=\mu \Delta u+(\lambda+\mu) \nabla \operatorname{div} u,
$$

which is obtained from the Gâteaux-derivative of $\mathcal{E}[\varphi][6]$. The Gâteaux derivatives of the energy terms yield the force terms $f_{\mathcal{D}}[6]$ and $f_{\mathcal{I}}[5]$.

The updates of $\varphi$ and the force estimation are decoupled: first, the forces are computed, then Eqn. 11 and 12 are solved treating the forces as constants. When combining this scheme with the alternating inverse consistency Eqn. 4, the resulting algorithm for a given iteration $k$ is

1. compute $f_{\mathcal{D}}\left[I_{1}, I_{2} ; \varphi^{k}\right]$ and $f_{\mathcal{I}}\left[\psi^{k} ; \varphi^{k}\right]$

2. compute $f_{\mathcal{D}}\left[I_{2}, I_{1} ; \psi^{k}\right]$ and $f_{\mathcal{I}}\left[\varphi^{k} ; \psi^{k}\right]$

3. solve Eqn. 11 for $\varphi^{k+1}$

4. solve Eqn. 12 for $\psi^{k+1}$

The fixed point iterations Eqn. 11 and Eqn. 12 are solved using the full multigrid strategy and red-black Gauss-Seidel relaxation [7] on a Gaussian image pyramid [1]. Fixed edges and bending side walls are used as border conditions [6]. The elasticity parameters are chosen such that Poisson's coefficient is zero, hence maximizing compressibility to allow compensation of local model inadequacies. Young's modulus is interpreted as a free variable in function of Poisson's coefficient and the PDE discretization $\Delta t$ since it has no physical meaning in image registration. The forces are capped to a maximum length which makes it possible to control the maximum contributions per iteration to the displacement field via $\Delta t$. Limiting the contributions to 0.5 voxel side lengths (Shannon theorem) ensures that the algorithm does not 'jump' over intensity barriers during optimization.

\section{EXPERIMENTS}

The framework was validated on 278 registrations of 295 US volumes from 17 patients. The 17 reference images were acquired shortly before the intervention, and the tracking images were acquired after a biopsy shot. The clinical protocol was approved by the ethical committee of the Pitié Salpétrière hospital, Paris, France, and all patients consented to participate to the study. The images were acquired with a GE Voluson and a RIC5-9 endorectal US probe. The algorithms were executed on a 4 -core $2.6 \mathrm{Ghz}$ processor. In order to provide a reference gold standard for the evaluation of registration accuracy, experts manually segmented 467 point fiducials that were clearly identifiable on multiple images (e.g. calcifications and cysts). The distances between fiducial pairs were measured after registration to estimate the local accuracy. Note that the unavoidable segmentation error increases the measured error in average; this approach hence underestimates accuracy. Accuracy was computed for all registrations that were qualified as valid by experts after visual inspection, which represent $97.8 \%$ of the registrations. The results for both rigid and elastic registration are given in Tab. 1, and a visual illustration of the registration performance is given in Fig. 1. 


\begin{tabular}{c|cccc} 
stage & $\begin{array}{c}\text { mean } \\
\text { distance }\end{array}$ & $\begin{array}{c}\text { standard } \\
\text { deviation }\end{array}$ & $\begin{array}{c}\text { max } \\
\text { distance }\end{array}$ & $\begin{array}{c}\text { execution } \\
\text { time }\end{array}$ \\
\hline unreg. & $13.76 \mathrm{~mm}$ & $7.89 \mathrm{~mm}$ & $51.61 \mathrm{~mm}$ & - \\
rigid & $1.33 \mathrm{~mm}$ & $0.85 \mathrm{~mm}$ & $4.19 \mathrm{~mm}$ & $2.1 \mathrm{~s}$ \\
elastic & $0.83 \mathrm{~mm}$ & $0.54 \mathrm{~mm}$ & $4.14 \mathrm{~mm}$ & $6.8 \mathrm{~s}$
\end{tabular}

Table 1. Accuracy study.

\section{DISCUSSION AND CONCLUSION}

Deformation estimation yields an overall accuracy of at least $0.83 \pm 0.54 \mathrm{~mm}$ on real patient data. This corresponds to an error reduction of $40 \%$ when compared to rigid 3D-3D registration. The average computation time of the registration was only $6.8 \mathrm{~s}$. We are confident that the algorithm can be accelerated to below $1 \mathrm{~s}$ on the same machine with simple optimization and parallelisation techniques, which is sufficient for assisted needle placement. With specialized standard hardware (GPUs), at least $5 \mathrm{~Hz}$ should be feasible.

The presented system does neither add complexity to the existing clinical protocol for prostate biopsies, nor does it change sterilization and working space requirements. No US beam tracking system is needed, hence reducing hardware requirements. The system recovers completely from patient movements without requiring any reinitialization, even if the patient is completely repositioned on the table during the intervention. We estimate that such significant pelvis movements occur in 15 to $25 \%$ of the interventions. Usage of 3D US instead of freehand 2.5 US considerably reduces the acquisition time of the reference volume, hence reducing patient movement artefacts and the total intervention time. Also, 3D US makes reliable deformation estimation possible.

Biopsy tracking systems potentially add significant clinical value to prostate cancer diagnosis and therapy planning. Immediate advantages are the possibility to avoid resampling of already biopsied tissues when repeating a biopsy series, interventional quality control of the biopsy distribution (e.g. detection of unsampled areas) and computer-assisted guidance to non-systematic targets. The latter could for example be identified on MR/spectroMR images of the gland. Moreover, the improved knowledge about the biopsy and thus the cancer position could be used to implement focal therapy strategies for prostate cancer. 3D US based elastic tracking can provide the precision required for such therapeutic applications.

\section{REFERENCES}

[1] M. Baumann, P. Mozer, V. Daanen, and J. Troccaz, "Towards 3D ultrasound image based soft tissue tracking: A transrectal ultrasound prostate image alignment system.," 2007, vol. 4792, pp. 26-33, Springer, LNCS.

[2] S. Xu, J. Krucker, P. Guion, N. Glossop, Z. Neeman, P. Choyke, A. K. Singh, and B. J. Wood, "Closed-

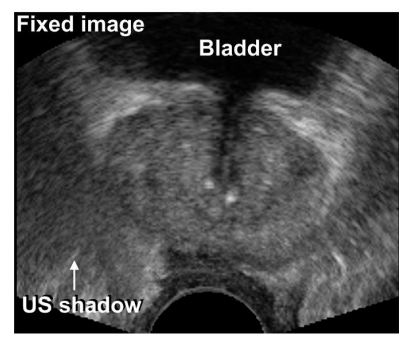

(a)

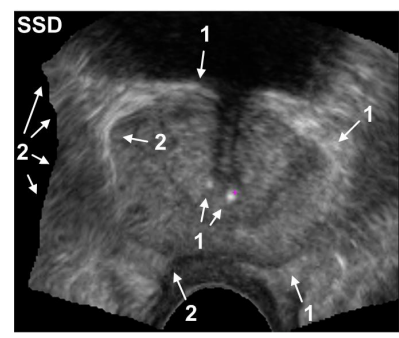

(c)

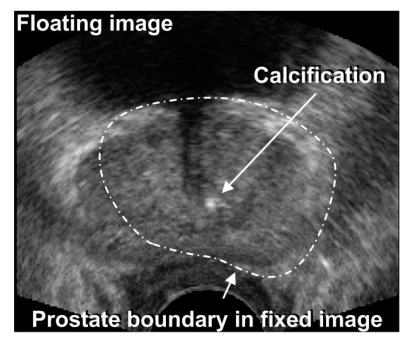

(b)

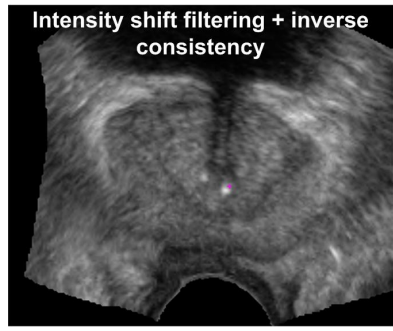

(d)
Fig. 1. Fig. (a) shows the fixed image with an ultrasound shadow and (b) the floating image. Fig. (c) shows the SSD registration, which yields correct results for part of the gland (1), but unnatural compressions in the shadow zone with a rough, oscillating displacement field (2). The intensity shift corrected, inverse consistent registration (d) is smooth and physically plausible (best viewed in PDF with zoom).

loop control in fused MR-TRUS image-guided prostate biopsy," MICCAI'07, vol. 4791, pp. 128-135, 2007.

[3] J. Bax, D. Cool, L. Gardi, K. Knight, D. Smith, J. Montreuil, S. Sherebrin, C. Romagnoli, and A. Fenster, "Mechanically assisted 3D ultrasound guided prostate biopsy system," Medical Physics, vol. 35, no. 12, pp. 53975410, December 2008.

[4] M. Baumann, A 3D ultrasound-based tracking system for prostate biopsy distribution quality insurance and guidance, Ph.D. thesis, Institut Polytechnique Grenoble, France, mai 2008.

[5] Z. Zhang, Y. Jiang, and H. Tsui, "Consistent multi-modal non-rigid registration based on a variational approach," Pattern Recognition Letters, pp. 715-725, 2006.

[6] Jan Modersitzki, Numerical Methods for Image Registration, Oxford University Press, 2004.

[7] W. Briggs, v. E. Henson, and S. F. McCormick, A multigrid tutorial, Soc for Industrial \& Applied Math, 2 edition, 2008. 